\title{
Psychiatric training of pre-registration house officers
}

\author{
J. M. O'Dwyer
}

\begin{abstract}
Aims and method The aim of the paper is to describe the pre-registration house officer training in psychiatry available in Sheffield. The report is the result of a postal questionnaire, involving all of those who undertook preregistration house officer training in psychiatry, since the inception of this option.

Results The results suggest that the psychiatry option as part of pre-registration house officer training was regarded as useful by almost all undertaking this training. Some of the suggestions to improve the experience are already incorporated into the current posts

Clinical implications The findings support the development of further posts of this type, and such developments may assist in overcoming some of the difficulties in recruitment into psychiatry as a career.
\end{abstract}

Pre-registration training is mandatory before full registration as a medical practitioner with the General Medical Council (General Medical Council, 1997). During the pre-registration year newly qualified doctors assume professional identities. Skills learned as students are tested and new skills acquired. The year usually consists of two periods of six months training and experience in each of medicine and surgery. However, this may be varied to four months in each of medicine and surgery and four months in another speciality, such as psychiatry or general practice.

Pre-registration training in Sheffield offers six months of medicine and six months of surgery. However, in about 1980, Professor C. P. Seagar (the then Professor of Psychiatry) introduced a further option of four months each of medicine. surgery and psychiatry, which is now offered in two posts. This allows six people per year to complete pre-registration training in psychiatry.

The aim of this report is to describe the career outcome of those undertaking this type of training experience, and to determine whether it was deemed a useful experience.

\section{The study}

Those who have undertaken pre-registration training in psychiatry were identified, contacted by post and a questionnaire regarding preregistration training in psychiatry completed. The questionnaire rated usefulness of the pre-registration posts in psychiatry on a scale of 1-10 (higher scores indicating that the posts were most useful), and collected information on the subsequent career. Those not replying were sent a reminder, and continued non-responders were excluded.

\section{Findings}

Eighty-six people were allocated psychiatry posts on the scheme. The total number of responses was $56(63 \%)$, including four with insufficient information and two who did not take the posts which were excluded. Therefore the number included and used for the report was 50 (58\% of the total sample).

Of those included the majority became general practitioners (48\%), followed by psychiatry (36\%). The remainder included public health medicine (4\%), surgical specialities (4\%), general medical posts $(4 \%)$ and others $(4 \%)$.

\section{Psychiatrists}

Eighteen of the fifty chose a career in psychiatry. Five were at consultant or equivalent grade in general adult psychiatry, learning disability psychiatry, child and adolescent psychiatry. rehabilitation psychiatry and psychotherapy. Four were at specialist registrar (SpR) or equivalent grade in psychogeriatrics, eating disorders, child and adolescent psychiatry and psychotherapy. Seven were senior house officers (SHOs), and two became staff grade doctors. The mean usefulness rating of pre-registration training in psychiatry was 7.4 for the consultants, 5.0 for SpRs, 7.3 for SHOs, and 6.5 for staff grade doctors. There was no significant difference between the groups.

Of those at consultant or SpR grade, five of the nine had no further psychiatric training in Sheffield. Of those who had further training in psychiatry in Sheffield, no one became a general adult psychiatrist. 


\section{General practice}

Of those in general practice, the ratings of how useful the pre-registration experience in psychiatry was showed an average rating of 7.5 (range 2-10). Only seven (29\%) did any further training in psychiatry. Their average usefulness of 7.7 compared with 7.4 for those not undertaking further training in psychiatry. The difference was not statistically significant.

\section{Others}

Other specialities included public health medicine (two) and surgical specialities (two), of which all were at SpR or consultant grade, and all reported the experience as useful. This group also included two people in medical posts, one doing a split post, one person pursuing further medical education, and one was not working. For this 'miscellaneous' group the mean usefulness rating was 7.8 , and the time from qualification was on average eight years. Only one had undertaken any further training in psychiatry.

Table 1 shows the usefulness rating of the preregistration house officer posts in psychiatry comparing the specialities. There was no significant difference between the groups.

Time from qualification varied from 19 to less than two years. The average time since qualification for those who replied was 10 years, for those in psychiatry it was 9.77 years, for those in general practice it was 10.5 years, and for the others eight years. Time from qualification had no relationship on the overall usefulness rating.

\section{Influence on the ultimate career choice}

Of those who were included, $58 \%$ felt that the pre-registration experience in psychiatry had influenced the ultimate career choice. Of those, $76 \%$ reported it as a positive experience. Positive comments included 'good clinical experience', 'development of communication skills', 'teamworking' and 'more useful than surgery'. A few found that they were unsuited to psychiatry (two), or had insufficient supervision (one). Of the 29 people whose career choice was influenced by the pre-registration posts in psychiatry. $55 \%$ were in psychiatry, $38 \%$ in general practice and $7 \%$ in other specialities.

Table 1. Usefulness of psychiatry pre-registration posts

\begin{tabular}{llll}
\hline Speciality & $\boldsymbol{n}$ & $\begin{array}{l}\text { Usefulness } \\
\text { (mean) }\end{array}$ & Range \\
\hline Psychiatry & 18 & 6.7 & $3-9$ \\
General practitioner & 24 & 7.5 & $2-10$ \\
Others & 7 & 7.8 & $7-9$ \\
Total & 50 & 7.2 & $2-10$ \\
\hline
\end{tabular}

\section{Identified problems}

The main problems identified included: isolation from peers (four); excessive anti-psychiatry (three); dependence on pre-registration house officers to deal with medical problems on psychiatry wards (two); lack of resuscitation training in psychiatry pre-registration house officer posts (two); lack of research training at pre-registration level (one); and lack of involvement in community care (one).

Suggested alterations to improve the training included; seeing out-patients (17) and regular consultant or SHO supervision (11). Some of these have been addressed, particularly supervision and resuscitation training, but the other issues need to be addressed.

\section{Comment}

The majority of the group undertaking preregistration house officer posts in psychiatry trained as general practitioners. For many this was the only psychiatric training undertaken. Almost all found the experience helpful in their subsequent careers. There is a need for further information regarding whether pre-registration house officer training in psychiatry renders those undertaking it more or less likely to have further psychiatric training. Clearly this experience should not replace that of the SHO. There were a few who having considered a career in psychiatry, altered their plans as a result of the pre-registration house officer training in psychiatry. Overall, however, the training was regarded as a very positive experience.

A proportion completed further training or are undertaking further training in psychiatry. Of note is the very small number of acute general psychiatrists at consultant grade. Almost all undertook training in the sub-specialities of psychiatry, with only one person having opted into general psychiatry at consultant level. The reason for this is unclear and needs to be investigated. Also, of those at SpR or consultant level, less than half continued psychiatry training locally. The reasons for this are unclear. As psychiatry is a speciality with a shortage, encouraging people to complete training in psychiatry should be a priority.

Training in psychiatry at pre-registration house officer grade was found useful in other specialities, including public health medicine and surgical specialities (orthopaedics and ophthalmology). The most useful aspect reported in such posts was training in communicating with patients and in acceptance of the patient as a whole rather than as a discrete disorder.

Clearly this report has several methodological limitations. The variable time since qualification will undoubtedly colour the results. People 
training further in psychiatry rated the usefulness of pre-registration training in psychiatry lower than their other colleagues. This could be explained by the notion that further training led to awareness of the limitations, and hence the training appears more limited. Further, the recall of events up to 19 years earlier could be coloured by subsequent events, or the skills learned could be developed as the career progresses. The data do not support these conclusions but more reports are needed.

Selection criteria for the posts may have resulted in those with a particular interest in psychiatry being selected for these posts. Trainees are selected by a process of trainees' choice and of those wanting this option selection follows interviews with the trainers. Therefore there is a degree of selection bias in applying these findings to the total pre-registration house officer group.

There are a number of suggestions which could be implemented to improve the posts. These include pre-registration house officers attending out-patient clinics with more senior clinicians and more direct supervision. Supervision is now mandatory, and occurs regularly. The other major problem is that of isolation from peers, as psychiatric units tend to be either in separate hospitals or on separate sites within the same hospital. This feeling of isolation could be remedied by ensuring that trainees have protected time to attend educational activities with their peers, and by appropriate induction programmes. Clearly the role of both the clinical tutor and mentor are also important.

\section{Reference}

GENERAL MEdicAL COUNCIL (1997) The New Doctor Recommendations on General Clinical Training made under Section 5 of the Medical Act 1983. London: GMC.

J. M. O'Dwyer, Senior Lecturer in Psychiatry, University of Sheffield Department of Psychiatry. Northern General Hospital, Herries Road. Sheffield S5 7AU 